\title{
Increased long-term mortality in heart failure due to sleep apnoea is not yet proven
}

\author{
T. Roebuck*, P. Solin*, D.M. Kaye", , P. Bergin", M. Bailey ${ }^{+}$, M.T. Naughton*
}

Increased long-term mortality in heart failure due to sleep apnoea is not yet proven. T. Roebuck, P. Solin, D.M. Kaye, P. Bergin, M. Bailey, M.T. Naughton. (C) ERS Journals Ltd 2004.

ABSTRACT: Previous small-scale studies of the effect of sleep-disordered breathing (SDB) on prognosis in congestive heart failure (CHF) are either lacking or conflicting.

The aim of this study was to assess the impact of the presence and type of SDB on mortality in a patient group with severe CHF referred to a specialised heart failure centre.

Out of 78 patients ((mean \pm SD) $53 \pm 9$ yrs, left ventricular ejection fraction $19.9 \pm 7.2 \%$ and pulmonary capillary wedge pressure $16.5 \pm 8.3 \mathrm{mmHg}$ ) followed-up over a median period of 52 months, $29 \%$ had no apnoea (CHF-N), 28\% had obstructive sleep apnoea (CHF-OSA) and 42\% had central sleep apnoea (CHF-CSA). At 52 months, their overall mortality was $40 \%$, and combined mortality and transplantation was $72 \%$. Mortality rates were similar between the three apnoea groups. Survivors had a similar prevalence of SDB $(71 \%)$ as the nonsurvivors $(70 \%)$. Although a significant increase in mortality was evident at 500 days in those patients with either CHF-SDB or CHF-CSA as compared with $\mathrm{CHF}-\mathrm{N}$, this was not significant at final follow-up (52 months) using Kaplan Meier analysis. Multivariate analysis identified transplantation but not SDB type or severity as a significant predictor of survival.

In conclusion, sleep-disordered breathing impacts upon early (500 day), but not longterm (52 month), mortality in a specialised heart failure centre. Eur Respir J 2004; 23: 735-740.
Depts of *Respiratory Medicine, ${ }^{\#}$ Cardiology, and ${ }^{+}$Epidemiology and Preventative Medicine, Alfred Hospital and Monash University, and Baker Heart Research Institute, Melbourne, Australia.

Correspondence: M.T. Naughton, Dept of Respiratory Medicine, Alfred Hospital, Commercial Road, Melbourne, Victoria, 3004, Australia.

Fax: 161392763601

E-mail: m.naughton@alfred.org.au

Keywords: Apnoea

heart failure

prognosis

\section{Received: May 302003}

Accepted after revision: December 122003

This study was supported by the Australian National Health and Medical Research Council.
In recent years, sleep-disordered breathing (SDB) has been identified as a common accompaniment in patients with congestive heart failure (CHF) [1]. Obstructive sleep apnoea (OSA), in which episodic upper airway collapse occurs in association with large negative intrathoracic pressures and hypoxaemia, has been described in $11-37 \%$ of $\mathrm{CHF}$ patients [2-4]. Central sleep apnoea (CSA), associated with a CheyneStokes pattern of respiration, is due to fluctuating heightened central respiratory drive and occurs in $33-42 \%$ of $\mathrm{CHF}$ patients [2-4].

It is unclear whether SDB of either type plays a role in the pathogenesis of CHF. In the case of OSA, reduced cardiac contractility and a tendency towards increased left ventricular mass has been described within 1-3 months of experimentally induced OSA in dogs [5]. In the largest cross-sectional study to date, SHAHAR et al. [6] reported an increased relative risk of self-reported heart failure in patients with OSA compared with those subjects without sleep apnoea. However, there are no data on the role of OSA on mortality in a $\mathrm{CHF}$ population.

In the case of CSA, hypoxaemia and arousals from sleep are thought to contribute to the increased mortality associated with CSA [4]. In 1985, FINDLEY et al. [7] reported 100\% mortality at 6 months in patients with Cheyne-Stokes respiration and $33 \%$ in those patients without Cheyne-Stokes respiration. Since 1991, three studies have shown increased rate of combined all-cause death and cardiac transplantation [8-10], while two have not $[11,12]$ (table 1).

In order to assess the impact of both apnoea types (CSA

For editorial comments see page 659. and OSA) on mortality in CHF, the current authors undertook a survival analysis of patients with symptomatic CHF who were being assessed for possible heart transplantation.

\section{Methods}

\section{Subjects}

Consecutive patients, aged 18-70 yrs, of either sex, with New York Heart Association (NYHA) dyspnoea class $\geqslant 2$, being assessed by the Alfred Hospital Heart Failure Service for transplantation consideration between June 1995 and May 1998 were enrolled if they met the following criteria: 1) clinical evidence of symptomatic CHF for at least 6 months duration and on medical therapy; 2) a left ventricular ejection fraction (LVEF) $\leqslant 55 \%$; and 3 ) stable condition, defined as no hospital admissions nor medication changes within the preceding 2 weeks. Exclusion criteria were significant neurological, respiratory or renal impairment, or morbid obesity $(>100 \mathrm{~kg})$. These patients were part of a study in which haemodynamics and SDB type were compared [4]. The Ethics Committee of the Alfred Hospital approved the study (no. 72/ 98) and patients, or next of kin, provided written informed consent.

\section{Sleep studies}

Overnight sleep studies were performed in the usual manner

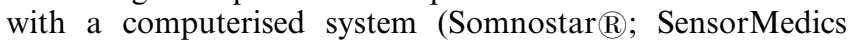


Table 1.-Comparison with other studies

\begin{tabular}{|c|c|c|c|c|c|c|}
\hline Author [first ref.] & HANLY [8] & ANDREAS [11] & TRAVERSI [12] & LANFRANCHI [9] & SIN [10] & Current study \\
\hline Population & NS & $\mathrm{CHF}$ unit & HTx unit & $\mathrm{CHF}$ unit & NS & HTx unit \\
\hline Subjects $n$ & 16 & 36 & 60 & 62 & 66 & 78 \\
\hline Follow-up months & 44 & 32 & 24 & 28 & 26 & 51 \\
\hline Mortality \% & $50(\mathrm{Tx})$ & 39 & 30 & $29(\mathrm{Tx})$ & 39 (Tx) & 40 \\
\hline CSA definition & Any & $\mathrm{TST}>20$ & Any & $\mathrm{AI}>30$ & AHI $>15$ & AHI $>5$ \\
\hline Age yrs & 64 & 54 & 55 & 57 & 59 & 53 \\
\hline $\mathrm{BMI} \mathrm{kg} \cdot \mathrm{m}^{-2}$ & 27 & 25 & NS & NS & NS & 27 \\
\hline Isch. $\%$ & 100 & 11 & 70 & 53 & 65 & 53 \\
\hline Female $\%$ & 0 & 16 & 3 & 14 & 10 & 22 \\
\hline LVEF \% & 23 & 20 & 23 & 23 & 20 & 20 \\
\hline Prognostic & Yes $(p=0.04)$ & No & No & Yes $(p=0.03)$ & Yes $(p=0.03)$ & No \\
\hline NYHA $2: 3: 4 \%$ & $0: 100: 0$ & NS & $33: 53: 13$ & NS & $\geqslant 3=40^{\#}$ & $28: 55: 17$ \\
\hline
\end{tabular}

CSA: central sleep apnoea; BMI: body mass index; Isch.: ischemic cardiomyopathy; LVEF: left ventricular ejection fraction; NYHA: New York Heart Association; NS: not stated; CHF: congestive heart failure; HTx: heart transplant; Tx: transplant; TST: total sleep time; AI: apnoea index; AHI: apnoea/hypopnoea index. ${ }^{\#}: 40 \%$ of patients had NYHA grades 3 or 4.

Corp, Yorba Linda, CA, USA) and sleep stages were manually scored according to standard criteria by an experienced scorer blinded to the patients' details, as previously described [4]. Electrocardiography and heart rate were recorded continuously from precordial lead II, arterial oxygen saturation was measured by an ear pulse oximeter $\left(\mathrm{Sp}, \mathrm{O}_{2}\right)$ (Fastrac; Sensormedics Corp) set at a 3-s averaging time. Chest and abdominal movements were monitored using respiratory effort bands calibrated for phase but not tidal volume (Resp-ez ${ }^{\text {TM}}$; EPM Systems, Midlothian, VA, USA). Oronasal airflow was monitored by thermocouples (ProTech Services, Woodinville, WA, USA) and snoring was monitored using a piezo snore sensor (ProTech Services).

A central apnoea was defined as an absence of oronasal airflow for $\geqslant 10 \mathrm{~s}$ associated with an absence of chest and abdominal movement. A central hypopnoea was defined as a reduction in oronasal airflow for $\geqslant 10 \mathrm{~s}$ associated with a $\geqslant 2 \%$ fall in $\mathrm{Sp}, \mathrm{O}_{2}$ with in-phase chest and abdominal movement, no increase in submental electromyogram (EMG) activity, and absence of snoring. An obstructive apnoea was defined as an absence of oronasal airflow for $\geqslant 10 \mathrm{~s}$ despite continued out-of-phase chest and abdominal movements. An obstructive hypopnoea was defined as a reduction in oronasal airflow for $\geqslant 10 \mathrm{~s}$ associated with a $\geqslant 2 \%$ fall in $\mathrm{Sp}, \mathrm{O}_{2}$ despite continued out-of-phase chest and abdominal movements, increased submental EMG activity or snoring. A mixed apnoea was defined, using the above criteria, when an apnoea was associated with an absence of effort followed by effort prior to initiation of ventilation. As upper airway closure is pathognomonic of mixed apnoeas, due to its relation to upper airway instability, these events were classified as obstructive events. The apnoea-hypopnoea index (AHI) was defined as the total number of apnoeas and hypopnoeas divided by the total sleep time, and expressed as the number of events per hour.

\section{Awake measurements}

Right heart pressures (pulmonary capillary wedge pressure (PCWP) and pulmonary arterial pressure) and cardiac index were measured within $12 \mathrm{~h}$ of the sleep study, supine, in the morning whilst awake, using a balloon-tipped flotation thermodilution catheter (7F Arrow; Arrow International, Reading, PA, USA) via the right internal jugular vein. LVEF was measured by ${ }^{99} \mathrm{Tc}$ radionuclide ventriculography using the equilibrium method, performed within 3 days of the overnight sleep study and urine collection. Arterial blood gas tensions were measured (Model 865; Ciba Corning Diagnostics
Corp., Medfield, MA, USA) in the CHF group, supine and awake, within $1 \mathrm{~h}$ prior to polysomnography.

\section{Protocol}

Patients were admitted for a 4-day assessment protocol. Sleep studies, LVEF and right heart catheter measurements were taken within $24 \mathrm{~h}$. Patient therapy was optimised following assessment.

Patients were divided into three groups, depending on the presence and type of sleep apnoea. Sleep apnoea was defined when AHI was $>5$ events $\cdot h^{-1}$, a threshold associated with an increased probability of CHF [6]. Patients with AHI $<5$ events $\cdot h^{-1}$ were defined as having no apnoeas (CHF-N group). CSA was defined when $\geqslant 85 \%$ of all apnoeas and hypopnoeas were purely central in origin, and central apnoeas and/or hypopnoeas occurred $>5$ events $\cdot h^{-1}$ (CHF-CSA group). Patients with an AHI $>5$ events $\cdot h^{-1}$, of which $<85 \%$ of events were typically central in type, were classified as CHF-OSA. Given the variance in CSA and OSA definition and classification between studies (table 1), patients were also grouped as AHI $>5$ events $\cdot h^{-1}$ of either type of SDB (central or obstructive) and were defined as CHF-SDB.

During the assessment for transplantation, all patients underwent further optimisation of medical therapy in the authors' specialised heart failure centre. This included one or more of the following: 1) regular telephone follow-up by a nurse practitioner; 2) attendance at a rehabilitation course; 3 ) further medication changes; 4) continuous positive airway pressure (CPAP; if AHI $>20$ events $\cdot h^{-1}$ and symptomatic) with $\mathrm{CHF}$ or supplemental oxygen if intolerant; and 5) cardiac transplantation.

Follow-up included checking hospital medical records, and telephone and written contact with the patients, the patients' family and general practitioners. Attempts were also made to ascertain the cause of death (sudden or progressive $\mathrm{CHF}$ or other).

\section{Statistical analysis}

The primary end-point for the study was overall survival [13]. The secondary end-point was transplantation-free survival. Comparisons of survival and transplant-free survival were made using analysis of variance across the three groups (CHF-N, CHF-OSA and CHF-CSA), and also between CHF-N and any apnoea type (CHF-SDB). In addition, comparisons of SDB severity and type were made between 
survivors and nonsurvivors using unpaired t-tests. Kaplan Meier plots were also used to assess the effect of SDB severity (AHI thresholds of $5,10,15$ or 20 events $\cdot \mathrm{h}^{-1}$ ) and type (central, obstructive or combined) on patient survival, as well as transplant-free survival. Survival was censored at the time of last contact. At the point of maximal divergence of survival curves (500 days), a further analysis was made using 95\% confidence intervals and differences assessed with t-tests. Finally, multivariate analyses were used to assess the effect of well-described cardiac and SDB parameters on survival status using Cox's Proportional Hazard Regression. A p-value of $<0.05$ was deemed significant and data were expressed as mean \pm SD.

\section{Results}

Seventy-eight patients (14 female) underwent initial assessment, at which time their mean \pm SD age was $53 \pm 9$ yrs, body mass index (BMI) $26.8 \pm 3.7 \mathrm{~kg} \cdot \mathrm{m}^{-2}$, LVEF 19.9 $\pm 7.2 \%$, PCWP $16.5 \pm 8.3 \mathrm{mmHg}$ and AHI $17.6 \pm 17.9$ events $\cdot h^{-1}$ (table 2 ). The ratio of ischaemic to idiopathic dilated cardiomyopathy was $53: 47 \%$, and NYHA classes 2, 3 and 4 were 28,55 and $17 \%$, respectively. At the time of assessment, all patients were taking angiotension converting enzyme inhibitors, $100 \%$ were on diuretics, $82 \%$ were on digoxin and $14 \%$ were on $\beta$-blockers. The median follow-up period was 52 months, at which time $60 \%$ of patients remained alive and $40 \%$ had undergone heart transplantation.

Out of the entire group of 78 patients, 23 had no SDB, 22 had CHF-OSA and 33 had CHF-CSA (table 1). The three groups were similar in terms of age, sex, BMI and LVEF. Lung function was similarly normal among the three groups and the PCWP was raised in the CHF-CSA group, as described previously [4]. The AHI was significantly lower in the CHF-N group and not statistically different between the CHF-CSA and CHF-OSA groups.

CPAP was trialled for up to 6 months in $26 \%$ of patients: $27 \%$ of the CHF-OSA group and $42 \%$ of the CHF-CSA group. Only $14 \%$ of the CHF-OSA and $12 \%$ of the CHF-CSA group continued CPAP for $>6$ months. Five and $6 \%$ of the

Table 2. - Patient characteristics

\begin{tabular}{lccccc}
\hline & Total & No SDB & OSA & CSA & p-value \\
\hline Subjects & 78 & 23 & 22 & 33 & \\
Age yrs & $53 \pm 8.5$ & $50 \pm 9$ & $53 \pm 9$ & $55 \pm 7.2$ & NS \\
F:M & $14: 64$ & $5: 18$ & $4: 18$ & $5: 28$ & \\
BMI kg $\cdot \mathrm{m}^{-2}$ & $26.8 \pm 3.7$ & $25.8 \pm 2.7$ & $28.4 \pm 4.3$ & $26.5 \pm 3.7$ & NS \\
Isch.:Idio. & $53: 47$ & $39: 61$ & $59: 41$ & $58: 42$ & \\
LVEF \% & $19.9 \pm 7.2$ & $21 \pm 8$ & $21 \pm 5$ & $19 \pm 8$ & NS \\
PCWP mmHg & $16.5 \pm 8.3$ & $11.5 \pm 7.1$ & $13.0 \pm 5.7$ & $22.4 \pm 7.1$ & $<0.01$ \\
AHI n h $^{-1}$ & $17.6 \pm 17.9$ & $2.1 \pm 1.6$ & $17.5 \pm 14.3$ & $28.5 \pm 18.4$ & $<0.001$ \\
Min. Sp, O $_{2} \%$ & $82.9 \pm 0.9$ & $86.2 \pm 0.9$ & $82.1 \pm 0.9$ & $80.5 \pm 0.9$ & $<0.001$ \\
NYHA 2:3:4 & $28: 55: 17$ & $34: 57: 9$ & $36: 55: 9$ & $18: 55: 27$ & \\
Tx \% & 40 & 39 & 36 & 42 & NS \\
Death \% & 40 & 39 & 36 & 42 & NS \\
Death post-Tx & $6 / 31$ & $2 / 9$ & $0 / 8$ & $4 / 14$ & NS \\
Tx + death & 72 & 70 & 73 & 73 & NS \\
Death & $55: 26: 19$ & $56: 33: 11$ & $50: 25: 25$ & $57: 21: 21$ & NS \\
\hline
\end{tabular}

SDB: sleep-disordered breathing; OSA: obstructive sleep apnoea; CSA: central sleep apnoea; F:M: female-to-male ratio; BMI: body mass index; Isch.: ischemic cardiomyopathy; Idio.: idiopathic cardiomyopathy; LVEF: left ventricular ejection fraction; PCWP: pulmonary capillary wedge pressure; AHI: apnoea/hypopnoea index; $\min . \mathrm{Sp}, \mathrm{O}_{2}$ : minimum arterial oxygen saturation as measured by pulse oximetry; NYHA: New York Heart Association; Tx: transplanted. \#: sudden/ progressive/other. NS: nonsignificant.
Table 3.-Comparison of survivors and nonsurvivors

\begin{tabular}{lccc}
\hline & Nonsurvivors & Survivors & p-value \\
\hline Subjects \% & 40 & 60 & \\
F:M & $7: 24$ & $7: 40$ & NS \\
Age yrs & $54.7 \pm 7.7$ & $52.1 \pm 9.0$ & NS \\
BMI kg.m ${ }^{-2}$ & $27.0 \pm 4.3$ & $26.7 \pm 3.3$ & NS \\
Isch.:Idio. \% & $61: 39$ & $47: 53$ & NS \\
NYHA 2:3:4 & $32: 52: 16$ & $53: 43: 4$ & NS \\
LVEF \% & $19.7 \pm 8.4$ & $20.0 \pm 6.4$ & NS \\
PCWP mmHg & $17.3 \pm 8.1$ & $16.0 \pm 8.5$ & NS \\
AHI $\cdot \mathrm{h}^{-1}$ & $17.7 \pm 16.2$ & $17.6 \pm 18.9$ & NS \\
Min. p, $\mathrm{O}_{2} \%$ & $83.2 \pm 0.7$ & $81.0 \pm 0.9$ & NS \\
SDB present $\%$ & 71 & 70 & NS \\
$\quad$ N $\%$ & 29 & 30 & NS \\
OSA $\%$ & 26 & 30 & NS \\
CSA $\%$ & 45 & 40 & NS \\
\hline
\end{tabular}

F: female; M: male; BMI: body mass index; Isch.: ischemic cardiomyopathy; Idio.: idiopathic cardiomyopathy; NYHA: New York Heart Association; LVEF: left ventricular ejection fraction; PCWP: pulmonary capillary wedge pressure; AHI: apnoea/hypopnoea index; $\min . \mathrm{Sp}, \mathrm{O}_{2}$ : minimum arterial oxygen saturation as measured by pulse oximetry; SDB: sleep-disordered breathing; N: no apnoea; OSA: obstructive sleep apnoea; CSA: central sleep apnoea. NS: nonsignificant.

CHF-OSA and CHF-CSA groups, respectively, were prescribed supplemental oxygen for home use.

The percentage of deaths, transplantations, and combined death and transplantation were not significantly different across the three groups (table 2). There was no difference in cardiac death due to either sudden or progressive CHF. The noncardiac causes of death were post-transplant rejection and sepsis $(n=4)$, renal failure $(n=1)$ and cancer $(n=1)$.

Comparison of the nonsurvivors with survivors (table 3 ) indicated no significant differences in any variable, apart from a tendency of more ischaemic cardiomyopathies in the nonsurvivor group. There were no differences in the presence or absence of SDB (either type) or each particular type.

The cumulative survival and transplant-free survival, based upon SDB type and status (figs 1-4), indicate that there was no significant impact of SDB upon survival. Re-analysis of survival curves based on CHF-SDB AHI thresholds of 10, 15 and 20 events $\cdot h^{-1}$ revealed no significant differences $(p=0.51$, 0.94 and 0.45 , respectively).

However, overall and transplant-free survival between $\mathrm{CHF}$ CSA and CHF-N groups (figs 1 and 3, respectively), and the CHF-SDB and CHF-N groups (figs 2 and 4, respectively) appeared to diverge maximally at the 500-day time-point. Moreover, total and transplant-free survival analysis at 500 days indicated a significant difference between CHF-N and the CHF-CSA groups (figs 1 and 3, respectively), but not between CHF-OSA and either CHF-N or CHF-CSA. Similarly, there was a significant difference in 500-day total and transplant-free survival between CHF-N and CHF-SDB groups (figs 2 and 4, respectively).

Using multivariate analyses, survival was significantly correlated with transplantation, whereas sex, age, BMI, CHF aetiology, NYHA, LVEF, PCWP, AHI, SDB type and SDB status were not.

\section{Discussion}

In this study, the largest and longest of its type, 78 patients with severe $\mathrm{CHF}$ being assessed for heart transplantation in a specialised heart failure centre were followed-up for a median period of 52 months. The presence, or type, of SDB was not significantly associated with increased mortality. For the first 


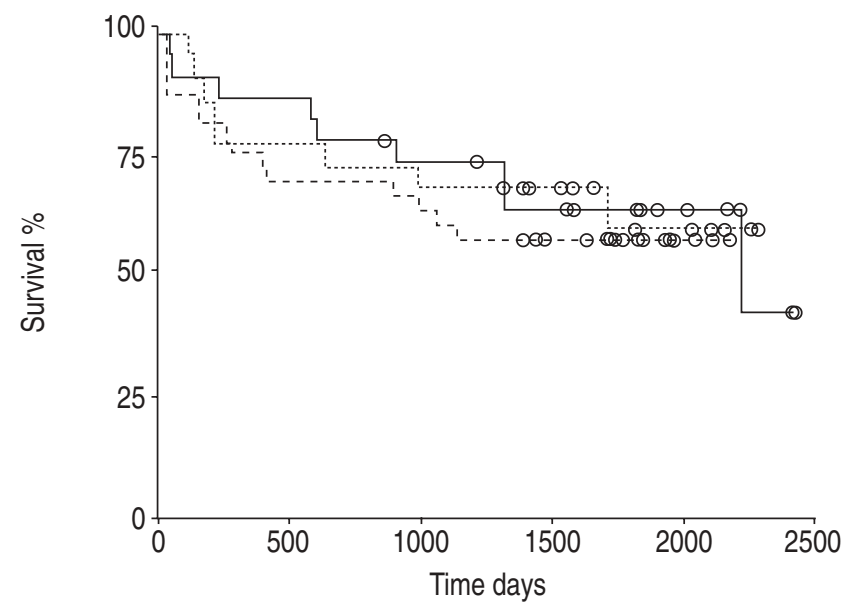

Fig. 1. - Impact of sleep disordered breathing type upon survival. - : CHF-N group, congestive heart failure no apnoea; $\cdots \cdots \cdot$. CHF-OSA group, congestive heart failure and obstructive sleep apnoea; -----: CHF-CSA group, congestive heart failure and central sleep apnoea. Note the reduced survival at 500 days in the CHF-CSA group (mean (95\% confidence interval) $0.70(0.54-0.86)$ ) as compared with the CHF-N group $(0.87(0.73-1.0) ; \mathrm{p}<0.05)$. The CHF-OSA group $(0.77$ $(0.59-0.95))$ was not statistically different to either CHF-N or CHFCSA groups at 500 days. Long-term mortality was no different between the three groups $(\mathrm{p}=0.78)$.

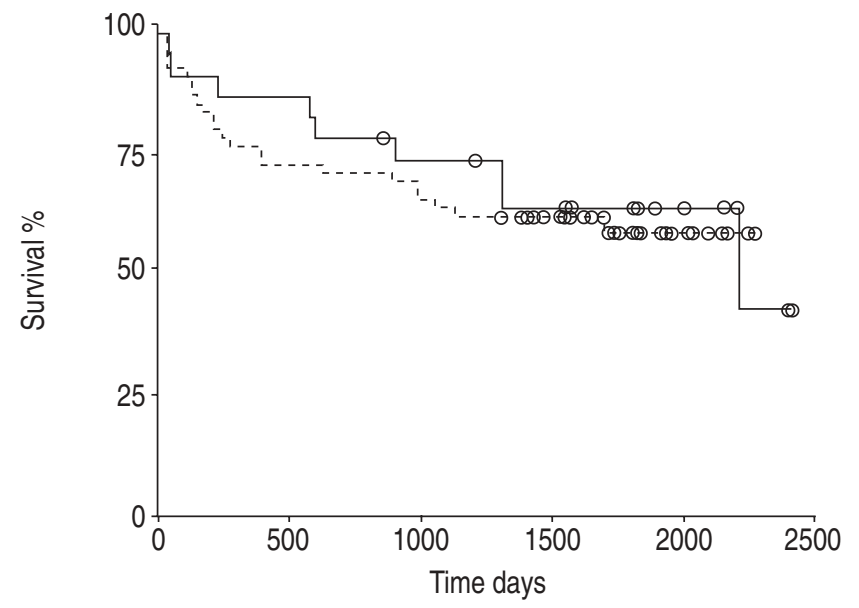

Fig. 2.-Impact of any sleep-disordered breathing upon overall survival. - : CHF-N, congestive heart failure no apnoea; -----: CHFSDB, congestive heart failure and any sleep-disordered breathing. At the 500-day time-point, the CHF-N group (mean ( $95 \%$ confidence interval) $(0.870 .73-0.10)$ had a significantly better survival than the CHF-SDB group (0.73 (0.61-0.85); $\mathrm{p}<0.05)$. However, long-term mortality was not significantly different between the two groups $(\mathrm{p}=0.73)$.

time, an analysis of OSA in CHF was undertaken and failed to show an effect upon mortality. Moreover, CSA was not found to be related to overall mortality in $\mathrm{CHF}$, which is both in agreement $[11,12]$ and conflict with previous studies [8-10].

Significant variations between the six mortality studies need to be highlighted (table 1). Most studies involved relatively small numbers of subjects (mean $<50$ patients) and followedup over a short period of time (mean 31 months). Statistical techniques varied considerably between the studies, as comparisons were made between either: 1) survivors versus nonsurvivors $[9,11]$; or 2) patients demonstrating central apnoea versus non-apnoeas [8-11]. Some studies undertook Kaplan Meier survival analyses [8-11] and only one undertook

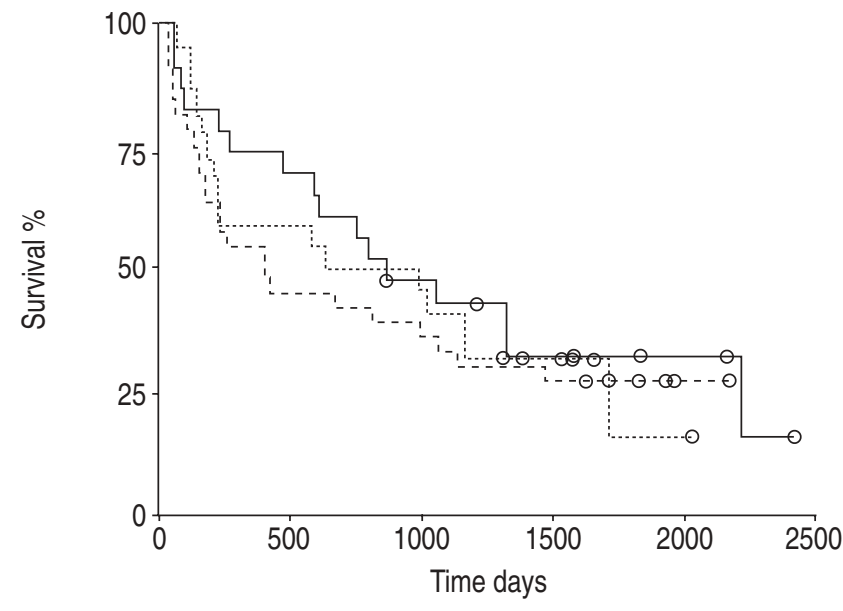

Fig. 3. - Impact of sleep-disordered breathing type on overall transplant-free survival. —: CHF-N group, congestive heart failure no apnoea; …. CHF-OSA, congestive heart failure and obstructive sleep apnoea; -----: CHF-CSA, congestive heart failure and central sleep apnoea. The 500-day survival in the CHF-N group (mean $(95 \%$ confidence interval) $0.70(0.50-0.90)$ ) was significantly better than the CHF-CSA group (0.45 (0.27-0.63); $\mathrm{p}<0.05)$, whereas the CHF-OSA group $(0.59(0.39-0.79))$ was not significantly different to either CHF$\mathrm{N}$ or CHF-CSA groups. The long-term mortality was not significantly different between the three groups $(\mathrm{p}=0.66)$.

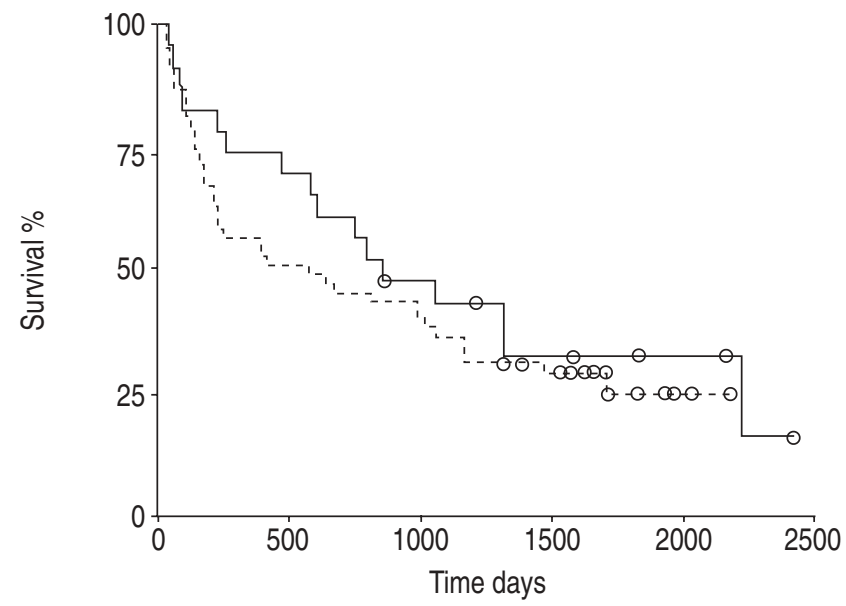

Fig. 4.-Impact of any sleep-disordered breathing upon overall transplant-free survival. - $\mathrm{CHF}-\mathrm{N}$, congestive heart failure no apnoea; -----: CHF-SDB, congestive heart failure and any sleepdisordered breathing. The 500-day survival in the CHF-N group (mean 95\% confidence interval) $0.70(0.50-0.90))$ was significantly better than the CHF-SDB group $(0.51(0.37-0.65) ; \mathrm{p}<0.05)$. However, long-term mortality was no different between the two groups $(\mathrm{p}=0.40)$.

multivariate analyses to assess confounding factors such as heart failure severity [12]. Definitions of survival varied, as some included transplantation combined with death of any cause as the primary end-point [8-10].

Patient selection varied between the six studies (table 1). The current study involved patients referred for transplantation assessment and treatment optimisation in a specialised heart failure centre. Such patients are generally selfmotivated, younger and less obese, and thus the present findings may not be applicable to the wider community of $\mathrm{CHF}$ patients. Some of the other studies involved a general cardiology population [8-10] and were older [8]. Indeed, the mean ages of subjects studied in the two negative studies [11, 
12] and the study presented here (53-55 yrs) are somewhat lower than the three positive studies (57-64 yrs) [8-10]. Despite this, however, no significant relationship was identified between age and survival. Despite the importance of obesity as a cause of CHF [14], some studies did not report the BMI of their patient population [8-10]. One study excluded patients with atrial fibrillation without reason [9].

The management of the patient group in the current study may have been more "aggressive" than in some of the other studies, which may have improved SDB [15], cardiac function and survival [16]. All previous mortality studies [8-12] did not describe additional medical therapies (apart from transplantation) initiated or altered during the period of observation. In the current study, all patients were offered an integrated approach to healthcare, which involved nurse practitioner contact, rehabilitation, further optimisation of medications and in $26 \%$ of patients the initiation of CPAP. Aggressive management of CHF may explain the change from significant higher early (500 day) to insignificant late (52 month) mortality in the CHF-CSA group compared with the CHF$\mathrm{N}$ group (figs 1 and 3 ).

The current authors have shown previously that optimisation of CHF management leads to a fall in both PCWP and CSA severity [4], and that successful cardiac transplantation leads to marked attenuation of CSA [17], suggesting that CHF and CSA severity move in parallel. Thus, it would be expected that optimisation of medical therapy would lead to reductions in severity of CSA. Unfortunately, follow-up sleep studies and optimisation of medical therapy were not performed following assessment in all subjects, which is similar to previous studies [8-12].

There is no consensus for an AHI threshold to initiate CPAP in CHF. In this study, a threshold of $>20$ events $\cdot h^{-1}$ with symptoms was chosen to initiate CPAP and CPAP was initiated in $26 \%$ of patients, half of whom continued on treatment beyond 6 months. There was no evidence of a survival benefit with CPAP, although this was not a randomised controlled trial. The precise threshold at which significant SDB occurs varied in the mortality studies [8-12] and in the randomised intervention studies of SDB with CPAP [18-20]. Moreover, whether the use of CPAP is sufficient to alter survival is not known. Indeed, CPAP has been shown to augment cardiac function in CSA [18] and OSA patients $[19,20]$, but there are scarce data concerning survival. In the study of SIN et al. [10], transplant-free survival at 36 months for CPAP-treated CHF-CSA and CHF-N groups was similar ( $\sim 68$ and $72 \%$, respectively). Whereas transplant-free survival at 36 months appeared to be greater in the non-CPAP-treated CHF-CSA $(\sim 60 \%)$ than the CHF-N group $(\sim 4 \%)$. In a recent trial of CPAP in CHF-OSA, CPAP was associated with two out of 19 deaths as compared with none out of 21 in the control group over 3 months [20]. Thus, larger studies are required to ascertain the impact of CPAP, or other therapies, upon SDB and survival in CHF.

The definitions of CSA, OSA and SDB differed significantly from study to study (table 1). In some studies, limited channel sleep studies rather than full polysomnography were performed [7, 9], thus the severity of SDB may have been erroneous. The present authors attempted to overcome this by looking at SDB type and combined severity separately (figs 1-4).

The limitations of this study, similar to all the previous studies, are that it fails to meet all the stringent requirements set out for survival studies [13], namely: 1) that subjects enter the trial at the same time in the course of their illness; 2) that there is a clearly defined and uniform end-point; 3) that a subject's chance of diagnosis and treatment is the same; and 4) that drop-outs should not be related to the outcome.

First, in this study, it could not be assessed whether patients entered the study at the same time course of their illness. This was, in part, due to the patients' inability to determine the accurate onset of their symptoms or failure of this to be accurately documented in the hospital records.

Secondly, the inclusion of transplantation as the primary end-point [8-10], or not [11, 12], may have influenced the results. Several factors influence the decision to perform a transplantation in a patient, such as age, physical fitness, blood group, associated illnesses, religion and social support, in addition to $\mathrm{CHF}$ severity. Moreover, persistence of CSA and development of OSA have been observed posttransplantation [17]. Accordingly, in the current study, the data were assessed with and without censoring the data at the time of transplantation and no effect of SDB was observed on survival or transplant-free survival.

Thirdly, the subjects' chance of diagnosis and treatment being the same is generally not possible given the variation in a patient's access to healthcare. In the current study, all patients were assessed by the same small group of heart failure specialist physicians who manage patients using a team approach. A limitation of the current study was that only $14 \%$ of patients were on $\beta$-blockers at the time of assessment, however, all patients were offered a trial of $\beta$-blockers during the period of optimisation. A further limitation of this study, common to the comparative studies [7-12], was that "optimisation" of medical therapies was not defined accurately, apart from transplantation and whether or not CPAP or supplemental oxygen were initiated. The present authors have, however, previously reported that improved cardiac function due to optimisation of medical therapy is associated with a reduction in CSA severity [4]. The current findings indicate that greater systematic attention to such medical changes need to be accurately documented in future studies.

In conclusion, an increased short-term (500 day), but not long-term (52 months), mortality was associated with central sleep apnoea in a group of 78 subjects with congestive heart failure attending a specialised heart failure centre over 52 months. Further long-term interventional studies are required to confirm whether sleep-disordered breathing has a significant impact upon cardiac function and survival [21].

\begin{abstract}
Acknowledgments. The authors would like to thank the staff of the Alfred Hospital Sleep Laboratory and the Heart Centre and Heart Lung Transplantation Service, in addition to M. Abramson for his assistance during the initiation of the study.
\end{abstract}

\section{References}

1. Naughton MT, Bradley TD. Sleep apnea in congestive heart failure. Clin Chest Med 1998; 19: 99-113.

2. Javaheri S, Parker TJ, Liming JD, et al. Sleep apnea in 81 ambulatory male patients with stable heart failure. Types and their prevalences, consequences and presentations. Circulation 1998; 97: 2154-2159.

3. Sin DD, Fitzgerald F, Parker JD, Newton G, Floras JS, Bradley TD. Risk factors for central and obstructive sleep apnea in 450 men and women with congestive heart failure. Am J Respir Crit Care Med 1999; 160: 1101-1106.

4. Solin P, Bergin P, Richardson M, Kaye DM, Walters EH, Naughton MT. Influence of pulmonary capillary wedge pressure on central apnea in heart failure. Circulation 1999; 99: 1574-1579.

5. Parker JD, Brooks D, Kozar LF, et al. Acute and chronic effects of airway obstruction on canine left ventricular performance. Am J Respir Crit Care Med 1999; 160: 18881896.

6. Shahar E, Whitney CW, Redline S, et al. Sleep disordered 
breathing and cardiovascular disease. Cross sectional results of the sleep heart health study. Am J Respir Crit Care Med 2001; 163: 19-25.

7. Findley LJ, Zwillich CW, Ancoli-Israel, Kripke D, Tisi G, Moser KM. Cheyne-Stokes breathing during sleep in patients with left ventricular heart failure. South Med $J$ 1985; 78: 11-15.

8. Hanly PJ, Zuberi-Khokhar NS. Increased mortality associated with Cheyne-Stokes respiration in patients with congestive heart failure. Am J Respir Crit Care Med 1996; 153: 272-276.

9. Lanfranchi PA, Braghiroli A, Bosimini E, et al. Prognostic value of nocturnal Cheyne-Stokes respiration in chronic heart failure. Circulation 1999; 99: 1435-1440.

10. Sin DD, Logan AG, Fitzgerald FS, Liu PP, Bradley TD. Effects of continuous positive airway pressure on cardiovascular outcomes in heart failure patients with and without Cheyne-Stokes respiration. Circulation 2000; 102: 61-66.

11. Andreas S, Hagenah G, Moller C, Werner GS, Kreuzer H. Cheyne-Stokes respiration and prognosis in congestive heart failure. Am J Cardiol 1996; 78: 1260-1264.

12. Traversi E, Callegari G, Pozzoli M, Opasich C, Tavazzi L. Sleep disorders and breathing alterations in patients with chronic heart failure. G Ital Cardiol 1997; 27: 423-429.

13. Norman GR, Steiner DL. PDQ Statistics $2^{\text {nd }}$ edition. Mosby, St Louis, 1997.

14. Kenchaiah S, Evans JE, Levy D, et al. Obesity and risk of heart failure. NEJM 2002; 347: 305-313.
15. Walsh JT, Andrews R, Starling R, et al. Effects of captopri and oxygen on sleep apnoea in patients with mild to moderate congestive heart failure. Heart 1995; 73: 237-241.

16. Stevenson LW, Warner SL, Steimle ARL, et al. The impending crisis awaiting cardiac transplantation: modeling a solution based upon selection. Circulation 1994; 89: 450457.

17. Mansfield DR, Solin P, Roebuck T, Bergin P, Kaye DM, Naughton MT. The effect of successful heart transplant treatment of heart failure on central sleep apnea. Chest 2003; 124: $1675-1681$

18. Naughton MT, Liu PP, Benard DC, Goldstein RS, Bradley TD. Treatment of congestive heart failure and CheyneStokes respiration during sleep by continuous positive airway pressure. Am J Respir Crit Care Med 1995; 151: 9297.

19. Kaneko Y, Floras JS, Usui K, et al. Cardiovascular effects of continuous positive airway pressure in patients with heart failure and obstructive sleep apnea. N Engl J Med 2003; 348: 1233-1241.

20. Mansfield DR, Gollogly NC, Bergin P, Kaye DM, Naughton MT. Controlled trial of continuous positive airway pressure in obstructive sleep apnea and heart failure. Am J Respir Crit Care Med 2004; 169: 361-366.

21. Bradley TD, Logan AG, Floras JF. Rationale and design of the Canadian Continuous Positive Airway Pressure Trial for Congestive Heart Failure patients with Central Sleep ApneaCANPAP. Can J Cardiol 2001; 1: 677-684. 